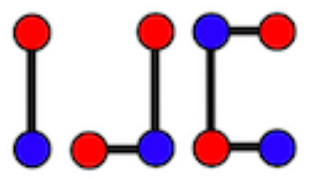

\title{
On $r$-dynamic coloring of some graph operations
}

\author{
Ika Hesti Agustin ${ }^{\mathrm{a}}$, Dafik ${ }^{\mathrm{b}}$, A. Y. Harsya ${ }^{\mathrm{a}}$ \\ a Department of Mathematics, University of Jember, Jember, Indonesia \\ ${ }^{b}$ Department of Mathematics Education, University of Jember, Jember, Indonesia \\ ikahestiagustin@gmail.com,d.dafik@unej.ac.id
}

\begin{abstract}
Let $G$ be a simple, connected and undirected graph. Given $r, k$ as any natural numbers. By an $r$-dynamic $k$-coloring of graph $G$, we mean a proper $k$-coloring $c(v)$ of $G$ such that $|c(N(v))| \geq$ $\min \{r, d(v)\}$ for each vertex $v$ in $V(G)$, where $N(v)$ is the neighborhood of $v$. The $r$-dynamic chromatic number, written as $\chi_{r}(G)$, is the minimum $k$ such that $G$ has an $r$-dynamic $k$-coloring. We note that the 1-dynamic chromatic number of graph is equal to its chromatic number, denoted by $\chi(G)$, and the 2-dynamic chromatic number of graph has been studied under the name a dynamic chromatic number, denoted by $\chi_{d}(G)$. By simple observation, we can show that $\chi_{r}(G) \leq \chi_{r+1}(G)$, however $\chi_{r+1}(G)-\chi_{r}(G)$ can be arbitrarily large, for example $\chi($ Petersen $)=$ $2, \chi_{d}($ Petersen $)=3$, but $\chi_{3}($ Petersen $)=10$. Thus, finding an exact values of $\chi_{r}(G)$ is not trivially easy. This paper will describe some exact values of $\chi_{r}(G)$ when $G$ is an operation of special graphs.
\end{abstract}

Keywords: $r$-dynamic coloring, $r$-dynamic chromatic number, graph operations

Mathematics Subject Classification : $05 \mathrm{C} 15$

\section{Introduction}

We refer all basic definition of graph to a handbook of graph theory written by Gross et. al [1]. Let $G=(V, E)$ be a simple, connected and undirected graph with vertex set $V$ and edge set $E$, and $d(v)$ be a degree of any $v \in V(G)$. The maximum degree and the minimum degree of $G$

Received: 12 July 2015, Revised: 25 July 2016, Accepted: 25 August 2016. 
are denoted by $\Delta(G)$ and $\delta(G)$, respectively. By a proper $k$-coloring of a graph $G$, we mean a map $c: V(G) \rightarrow S$, where $|S|=k$, such that any two adjacent vertices receive different colors. An $r$-dynamic $k$-coloring is a proper $k$-coloring $c$ of $G$ such that $|c(N(v))| \geq \min \{r, d(v)\}$ for each vertex $v$ in $V(G)$, where $N(v)$ is the neighborhood of $v$ and $c(S)=\{c(v): v \in S\}$ for a vertex subset $S$. The $r$-dynamic chromatic number, written as $\chi_{r}(G)$, is the minimum $k$ such that $G$ has an $r$-dynamic $k$-coloring. Note that the 1 -dynamic chromatic number of graph is equal to its chromatic number, denoted by $\chi(G)$, and the 2-dynamic chromatic number was introduced by Montgomery [5] under the name a dynamic chromatic number, denoted by $\chi_{d}(G)$. He conjectured $\chi_{2}(G) \leq \chi(G)+2$ when $G$ is regular, which remains open. Akbari et. al [4] proved Montgomery's conjecture for bipartite regular graphs. Lai, et.al [6] proved $\chi_{2}(G) \leq \Delta(G)+1$ when $\Delta(G) \geq 3$ and no component contains $C_{5}$. Kim et. al [3] proved $\chi_{2}(G) \leq 4$ when $G$ is planar and no component is $C_{5}$ and also $\chi_{d} \leq 5$ whenever $G$ is planar.

Obviously, $\chi(G) \leq \chi_{2}(G)$, but it was shown in [6] that the difference between the chromatic number and the dynamic chromatic number can be arbitrarily large. However, it was conjectured that for regular graphs the difference is at most 2. Some properties of dynamic coloring were studied in [3, 4, 6]. It was proved in [8] that, for a connected graph $G$, if $\Delta(G) \leq 3$, then $\chi_{2}(G) \leq 4$ unless $G=C_{5}$, in which case $\chi_{2}\left(C_{5}\right)=5$ and if $\Delta(G) \geq 4$ then $\chi(G) \leq \Delta+1$. Considering those results, finding an exact value of $\chi_{r}(G)$ is significantly useful as there are a little number of results provide an exact value of $\chi_{r}(G)$. Thus, in this paper we will show it when $G$ is an operation of special graphs.

\section{Some Useful Theorem}

The following Theorem are useful for determining the dynamic coloring of graphs. Jahanbekam et. al [7] characterize the upper bound of $\chi_{r}(G)$ in term of the diameter of graph.

Theorem 1.1. [7] If diam $(G)=2$, then $\chi_{2}(G) \leq \chi(G)+2$, with equality holds only when $G$ is a complete bipartite graph or $C_{5}$.

Theorem 1.2. [7] If $G$ is a $k$-chromatic graph with diameter at most 3 , then $\chi_{2}(G) \leq 3 k$, and this bound is sharp when $k \geq 2$.

In term of the maximum degree of graph, the $r$-dynamic of graph satisfies as follows

Observation 1. [7] $\chi_{r}(G) \geq \min \{\Delta(G), r\}+1$, and this is sharp. If $\Delta(G) \leq r$ then $\chi_{r}(G)=$ $\min \{\Delta(G), r\}$.

Theorem 1.3. [7] $\chi_{r}(G) \leq r \Delta(G)+1$, with equality for $r \geq 2$ if and only if $G$ is $r$-regular with diameter 2 and girth 5 .

The last for the graph operations, Jahanbekam et. al proved the following theorem.

Theorem 1.4. [7] If $\delta(G) \geq r$ then $\chi_{r}(G \square H)=\max \{\chi(G), \chi(H)\}$. 


\section{The Results}

Now, we are ready to show our results on $r$-dynamic coloring for some special graph operations. Apart from showing the $r$-dynamic chromatic number we also show the colors $c(v \in V(G))$ for clarity. Some graph operations which have been found in this paper are $P_{n}+C_{m}, C_{n} \square S_{m}, C_{n} \otimes$ $S_{m}, C_{n}\left[S_{m}\right], C_{n} \odot S_{m}, \operatorname{shack}\left(P_{n} \square C_{m}, v, s\right)$, $\operatorname{amal}\left(P_{n} \square C_{m}, v, s\right)$.

Theorem 1.5. Let $G$ be a joint $P_{n}$ and $C_{m}$. For $n \geq 2$ dan $m \geq 3$, the r-dynamic chromatic number of $G$ is

$$
\begin{gathered}
\chi\left(P_{n}+C_{m}\right)=\chi_{d}\left(P_{n}+C_{m}\right)=\chi_{3}\left(P_{n}+C_{m}\right)= \begin{cases}4, & \text { for } m \text { even } \\
5, & \text { for } m \text { odd }\end{cases} \\
\chi_{4}\left(P_{n}+C_{m}\right)= \begin{cases}5, & \text { for } m \equiv 3(\bmod 3) \\
6, & \text { otherwise }\end{cases}
\end{gathered}
$$

Proof. The graph $P_{n}+C_{m}$ is a connected graph with vertex set $V\left(P_{n}+C_{m}\right)=\left\{x_{i} ; 1 \leq i \leq n\right\} \cup$ $\left\{y_{j} ; 1 \leq j \leq m\right\}$ and $E\left(P_{n}+C_{m}\right)=\left\{x_{i} x_{i+1} ; 1 \leq i \leq n-1\right\} \cup\left\{y_{j} y_{j+1}, y_{m} y_{1} ; 1 \leq j \leq m-1\right\}$ $\cup\left\{x_{i} y_{j} ; 1 \leq i \leq n ; 1 \leq j \leq m\right\}$. Thus $p=\left|V\left(P_{n}+C_{m}\right)\right|=n+m, q=|E(G)|=n m+n+m-1$ and $\Delta\left(P_{n}+C_{m}\right)=m+2$. By Observation 1, the lower bound of $r$-dynamic chromatic number $\chi_{r}\left(P_{n}+C_{m}\right) \geq \min \left\{\Delta\left(P_{n}+C_{m}\right), r\right\}+1=\{m+2, r\}+1$.

For $\chi\left(P_{n}+C_{m}\right)=\chi_{d}\left(P_{n}+C_{m}\right)=\chi_{3}\left(P_{n}+C_{m}\right)$, define the vertex colouring $c: V\left(P_{n}+C_{m}\right) \rightarrow$ $\{1,2, \ldots, k\}$ for $n \geq 2$ and $m \geq 3$ as follows:

$$
\begin{aligned}
& c\left(x_{i}\right)=\left\{\begin{array}{l}
1,1 \leq i \leq n, i \text { odd } \\
2,1 \leq i \leq n, i \text { even }
\end{array} \quad c\left(y_{j}\right)=\left\{\begin{array}{l}
3,1 \leq j \leq m, j \text { odd, } m \text { even } \\
4,1 \leq j \leq m, j \text { even, } m \text { even }
\end{array}\right.\right. \\
& c\left(y_{j}\right)=\left\{\begin{array}{l}
3,1 \leq j \leq m-1, j \text { odd, } m \text { odd } \\
4,1 \leq j \leq m-2, j \text { even, } m \text { odd } \\
5, j=m, m \text { odd }
\end{array}\right.
\end{aligned}
$$

It is easy to see that $c: V\left(P_{n}+C_{m}\right) \rightarrow\{1,2, \ldots, 4\}$ and $c: V\left(P_{n}+C_{m}\right) \rightarrow\{1,2, \ldots, 5\}$, for $m$ even and odd respectively, are proper coloring. Thus, $\chi\left(P_{n}+C_{m}\right)=4$ and $\chi\left(P_{n}+C_{m}\right)=5$, for $m$ even and odd respectively. By definition, since $\min \left\{|c(N(v))|\right.$, for every $\left.v \in V\left(P_{n}+C_{m}\right)\right\}=$ $3 \leq \delta\left(P_{n}+C_{m}\right)=4$, it implies $\chi\left(P_{n}+C_{m}\right)=\chi_{d}\left(P_{n}+C_{m}\right)=\chi_{3}\left(P_{n}+C_{m}\right)$.

For $\chi_{4}\left(P_{n}+C_{m}\right)$, define the vertex colouring $c: V\left(P_{n}+C_{m}\right) \rightarrow\{1,2, \ldots, k\}$ for $n \geq 2$ and $m \geq 3$ as follows:

For $m \equiv 3(\bmod 3)$

$$
\begin{gathered}
c\left(x_{i}\right)=\left\{\begin{array}{r}
1,1 \leq i \leq n, i \text { odd } \\
2,1 \leq i \leq n, i \text { even }
\end{array}\right. \\
c\left(y_{j}\right)=\left\{\begin{array}{r}
3,1 \leq j \leq m, j \equiv 5(\bmod 3) \\
4,1 \leq j \leq m, j \equiv 4(\bmod 3) \\
5,1 \leq j \leq m, j \equiv 3(\bmod 3)
\end{array}\right.
\end{gathered}
$$


For $m \equiv 4(\bmod 3)$

$$
\begin{gathered}
c\left(x_{i}\right)=\left\{\begin{array}{l}
1,1 \leq i \leq n, i \text { odd } \\
2,1 \leq i \leq n, i \text { even }
\end{array}\right. \\
c\left(y_{j}\right)=\left\{\begin{array}{l}
3,1 \leq j \leq m-1, j \equiv 5(\bmod 3) \\
4,1 \leq j \leq m-1, j \equiv 4(\bmod 3) \\
5,1 \leq j \leq m-1, j \equiv 3(\bmod 3) \\
6, j=m
\end{array}\right.
\end{gathered}
$$

For $m \equiv 5(\bmod 3)$

$$
\begin{gathered}
c\left(x_{i}\right)=\left\{\begin{array}{l}
1,1 \leq i \leq n, i \equiv 5(\bmod 3) \\
2,1 \leq i \leq n, i \equiv 4(\bmod 3) \\
3,1 \leq i \leq n, i \equiv 3(\bmod 3)
\end{array}\right. \\
c\left(y_{j}\right)=\left\{\begin{array}{l}
4,1 \leq j \leq m, j \text { odd }, m \text { even } \\
5,1 \leq j \leq m, j \text { even } m \text { even }
\end{array}\right. \\
c\left(y_{j}\right)=\left\{\begin{array}{l}
4,1 \leq j \leq m-1, j \text { odd }, m \text { odd } \\
5,1 \leq j \leq m-2, j \text { even } m \text { odd } \\
6, j=m, m \text { odd }
\end{array}\right.
\end{gathered}
$$

It is easy to see, for $m \equiv 3(\bmod 3) c: V\left(P_{n}+C_{m}\right) \rightarrow\{1,2, \ldots, 5\}$, and otherwise $c:$ $V\left(P_{n}+C_{m}\right) \rightarrow\{1,2, \ldots, 6\}$ are proper coloring. Thus, for $m \equiv 3(\bmod 3), \chi_{4}\left(P_{n}+C_{m}\right)=5$ and $\chi\left(P_{n}+C_{m}\right)=6$ otherwise. By definition, since $\min \left\{|c(N(v))|\right.$, for every $\left.v \in V\left(P_{n}+C_{m}\right)\right\}=$ $4 \leq \delta\left(P_{n}+C_{m}\right)=4$, it is proved that $\chi_{4}\left(P_{n}+C_{m}\right)=5$.

Problem 1. Let $G$ be a joint $P_{n}$ and $C_{m}$. For $n \geq 2$ and $m \geq 3$, determine the $r$-dynamic chromatic number of $G$ when $r \geq 5$.

Theorem 1.6. Let $G$ be a joint $W_{n}$ and $P_{m}$. For $n \geq 3$ dan $m \geq 2$, the $r$-dynamic chromatic number of $G$ is

$$
\chi(G)=\chi_{d}(G)=\chi_{3}(G)=\chi_{4}(G)\left\{\begin{array}{l}
5, \text { for } n \text { even } \\
6, \text { for } n \text { odd }
\end{array}\right.
$$

Proof. The graph $W_{n}+P_{m}$ is a connected graph with vertex set $V\left(W_{n}+P_{m}\right)=\left\{A, x_{i}, y_{j} ; 1 \leq\right.$ $i \leq n ; 1 \leq j \leq m\}$ and $E\left(P_{n}+C_{m}\right)=\left\{A x_{i} ; 1 \leq i \leq n\right\} \cup\left\{x_{i} x_{i+1} ; 1 \leq i \leq n-1\right\} \cup\left\{x_{1} x_{n}\right\}$ $\cup\left\{A y_{j} ; 1 \leq j \leq m\right\} \cup\left\{x_{i} y_{j} ; 1 \leq i \leq n-1 ; 1 \leq j \leq m\right\} \cup\left\{x_{n} y_{j} ; 1 \leq j \leq m\right\} \cup\left\{y_{j} y_{j+1} ; 1 \leq\right.$ $j \leq m-1\}$. Thus $p=\left|V\left(W_{n}+P_{m}\right)\right|=n+m+1, q=|E(G)|=n m+2 n+2 m-1$ and $\Delta\left(W_{n}+P_{m}\right)=m+n$. By Observation 1, the lower bound of $r$-dynamic chromatic number $\chi_{r}\left(W_{n}+P_{m}\right) \geq \min \left\{\Delta\left(W_{n}+P_{m}\right), r\right\}+1=\{m+n, r\}+1$. Define the vertex coloring $c: V\left(W_{n}+P_{m}\right) \rightarrow\{1,2, \ldots, k\}$ for $n \geq 3$ and $m \geq 2$ as follows:

For $n$ even

$$
c\left(x_{i}\right)=\left\{\begin{array}{l}
1,1 \leq i \leq n, i \text { even } \\
2,1 \leq i \leq n, i \text { odd }
\end{array} \quad c\left(y_{j}\right)=\left\{\begin{array}{l}
4,1 \leq j \leq m, j \text { odd } \\
5,1 \leq j \leq m, j \text { even }
\end{array}\right.\right.
$$


For $n$ odd

$$
c\left(x_{i}\right)=\left\{\begin{array}{l}
1,1 \leq i \leq n-1, i \text { odd } \\
2,1 \leq i \leq n-1, i \text { even } \\
4, i=n
\end{array} \quad c\left(y_{j}\right)=\left\{\begin{array}{l}
5,1 \leq j \leq m, j \text { odd } \\
6,1 \leq j \leq m, j \text { even }
\end{array}\right.\right.
$$

It is easy to see that $c: V\left(W_{n}+P_{m}\right) \rightarrow\{1,2, \ldots, 4\}$ and $c: V\left(W_{n}+P_{m}\right) \rightarrow\{1,2, \ldots, 5\}$, for $n$ even and odd respectively, is proper coloring. Thus, $\chi\left(W_{n}+P_{m}\right)=5$ and $\chi\left(W_{n}+P_{m}\right)=6$, for $m$ even and odd respectively. By definition, since $\min \left\{|c(N(v))|\right.$, for every $\left.v \in V\left(W_{n}+P_{m}\right)\right\}=4$, it implies $\chi\left(W_{n}+P_{m}\right)=\chi_{d}\left(W_{n}+P_{m}\right)=\chi_{3}\left(W_{n}+P_{m}\right)=\chi_{4}\left(W_{n}+P_{m}\right)$. It completes the proof.

Problem 2. Let $G$ be a joint $W_{n}$ and $P_{m}$. For $n \geq 2$ and $m \geq 3$, determine the $r$-dynamic chromatic number of $G$ when $r \geq 5$.

Theorem 1.7. Let $G$ be a composition of graph $C_{n}$ on $S_{m}$. For $n \geq 3$ dan $m \geq 3$, the $r$-dynamic chromatic number of $G$ is

$$
\chi\left(C_{n}\left[S_{m}\right]\right)=\chi_{d}\left(C_{n}\left[S_{m}\right]\right)=\chi_{3}\left(C_{n}\left[S_{m}\right]\right)=\left\{\begin{array}{l}
4, \text { for } n \text { even } \\
6, \text { for } n \text { odd }
\end{array}\right.
$$

Proof. The graph $C_{n}\left[S_{m}\right]$ is a connected graph with vertex set $V\left(C_{n}\left[S_{m}\right]\right)=\left\{A_{i} ; 1 \leq i \leq n\right\}$ $\cup\left\{x_{i, j} ; 1 \leq i \leq n 1 \leq j \leq m\right\}$ and $E\left(C_{n}\left[S_{m}\right]\right)=\left\{A_{i} A_{i+1} ; 1 \leq i \leq n-1\right\} \cup\left\{A_{n} A_{1}\right\}$ $\cup\left\{x_{i, j} x_{i+1, j} ; 1 \leq i \leq n-1 ; 1 \leq j \leq m\right\} \cup\left\{x_{n, j} x_{1, j} ; 1 \leq j \leq m\right\} \cup\left\{A_{i} x_{i, j} ; 1 \leq i \leq\right.$ $n ; 1 \leq j \leq m\} \cup\left\{A_{i} x_{i+1, j} ; 1 \leq i \leq n-1 ; 1 \leq j \leq m\right\} \cup\left\{A_{i} x_{i-1, j} ; 2 \leq i \leq n ; 1 \leq j \leq m\right\}$ $\cup\left\{A_{1} x_{n, j} ; 1 \leq j \leq m\right\} \cup\left\{A_{n} x_{1, j} ; 1 \leq j \leq m\right\}$. Thus $\left|V\left(C_{n}\left[S_{m}\right]\right)\right|=n m+n$ and $\left|E\left(C_{n}\left[S_{m}\right]\right)\right|=$ $4 n m+n$ and $\Delta\left(C_{n}\left[S_{m}\right]\right)=3 m+2$. By Observation 1, the lower bound of $r$-dynamic chromatic number $\chi_{r}\left(C_{n}\left[S_{m}\right]\right) \geq \min \left\{\Delta\left(C_{n}\left[S_{m}\right]\right), r\right\}+1=\{3 m+2, r\}+1$. Define the vertex colouring $c: V\left(C_{n}\left[S_{m}\right]\right) \rightarrow\{1,2, \ldots, k\}$ for $n \geq 3$ and $m \geq 3$ as follows:

$$
\begin{gathered}
c\left(A_{i}\right)=\left\{\begin{array}{r}
1,1 \leq i \leq n, i \text { odd } \\
2,1 \leq i \leq n, i \text { even }
\end{array}\right. \\
c\left(x_{i, j}\right)=\left\{\begin{array}{r}
3,1 \leq i \leq n, i \text { odd } 1 \leq j \leq m \text { and } n \text { even } \\
4,1 \leq i \leq n, i \text { odd } 1 \leq j \leq m \text { and } n \text { even }
\end{array}\right. \\
c\left(x_{i, j}\right)=\left\{\begin{array}{l}
3,1 \leq i \leq n-2, i \text { odd } ; 1 \leq j \leq m \text { and } n \text { odd } \\
4,1 \leq i \leq n-1, i \text { odd } ; 1 \leq j \leq m \text { and } n \text { odd } \\
5, i=n
\end{array}\right.
\end{gathered}
$$

It is easy to see that $c: V\left(C_{n}\left[S_{m}\right]\right) \rightarrow\{1,2, \ldots, 4\}$ and $c: V\left(C_{n}\left[S_{m}\right]\right) \rightarrow\{1,2, \ldots, 5\}$, for $n$ even and odd respectively, is proper coloring. Thus, $\chi\left(C_{n}\left[S_{m}\right]\right)=4$ and $\chi\left(C_{n}\left[S_{m}\right]\right)=5$, for $n$ even and odd respectively. By definition, since $\min \{|c(N(v))|$,

for every $\left.v \in V\left(C_{n}\left[S_{m}\right]\right)\right\}=3 \leq \delta\left(C_{n}\left[S_{m}\right]\right)=5$, it implies $\chi\left(C_{n}\left[S_{m}\right]\right)=\chi_{d}\left(C_{n}\left[S_{m}\right]\right)=$ $\chi_{3}\left(C_{n}\left[S_{m}\right]\right)$. It completes the proof.

Problem 3. Let $G$ be a cartesian product of $C_{n}$ and $S_{m}$. For $n \geq 3$ and $m \geq 3$, determine the $r$-dynamic chromatic number of $G$ when $r \geq 4$. 
Theorem 1.8. Let $G$ be a crown product of $W_{n}$ on $P_{m}$. For $n \geq 3$ dan $m \geq 2$, the r-dynamic chromatic number of $G$ is

$$
\chi\left(W_{n} \odot P_{m}\right)=\chi_{d}\left(W_{n} \odot P_{m}\right)=\left\{\begin{array}{l}
3, \text { for } n \text { even } \\
4, \text { for } n \text { odd }
\end{array}\right.
$$

Proof. The graph $W_{n} \odot P_{m}$ is a connected graph with vertex set $V\left(W_{n} \odot P_{m}\right)=\left\{A, x_{i}, x_{i, j}, y_{j} ; 1 \leq\right.$ $i \leq n ; 1 \leq j \leq m\}$ and $E\left(W_{n} \odot P_{m}\right)=\left\{A x_{i} ; 1 \leq i \leq n\right\} \cup\left\{x_{i} x_{i+1} ; 1 \leq i \leq n-1\right\} \cup\left\{A y_{j} ; 1 \leq\right.$ $j \leq m\} \cup\left\{y_{j} y_{j+1} ; 1 \leq j \leq m-1\right\} \cup\left\{x_{1} x_{n}\right\} \cup\left\{x_{i} x_{i, j} ; 1 \leq i \leq n ; 1 \leq j \leq m\right\} \cup\left\{x_{i, j} x_{i, j+1} ; 1 \leq\right.$ $i \leq n ; 1 \leq j \leq m-1\}$. Thus $\left|V\left(W_{n}\left[P_{m}\right]\right)\right|=n m+n+m+1$ and $\left|E\left(W_{n} \odot P_{m}\right)\right|=$ $2 n m+n+2 m-1$ and $\Delta\left(W_{n} \odot P_{m}\right)=n+m$. By Observation 1, the lower bound of $r$-dynamic chromatic number $\chi_{r}\left(W_{n} \odot P_{m}\right) \geq \min \left\{\Delta\left(W_{n} \odot P_{m}\right), r\right\}+1=\{n+m, r\}+1$. Define the vertex coloring $c: V\left(W_{n} \odot P_{m}\right) \rightarrow\{1,2, \ldots, k\}$ for $n \geq 3$ and $m \geq 2$ as follows: $A=4$ and

$$
c\left(y_{j}\right)=\left\{\begin{array}{l}
1,1 \leq j \leq m, j \text { even } \\
3,1 \leq j \leq m, j \text { odd }
\end{array}\right.
$$

For $n$ even

$$
\begin{gathered}
c\left(x_{i, j}\right)=\left\{\begin{array}{l}
1,1 \leq i \leq n, i \text { odd } ; 1 \leq j \leq m, j \text { even } \\
2,1 \leq i \leq n, i \text { even } 1 \leq j \leq m, j \text { even } \\
3,1 \leq j \leq m, j \text { odd } 1 \leq i \leq n
\end{array}\right. \\
c\left(x_{i}\right)=\left\{\begin{array}{c}
1,1 \leq i \leq n, i \text { even } \\
2,1 \leq i \leq n, i \text { odd }
\end{array}\right.
\end{gathered}
$$

For $n$ odd

$$
\begin{gathered}
c\left(x_{i, j}\right)=\left\{\begin{array}{l}
1,1 \leq i \leq n, i \text { odd } 1 \leq j \leq m, j \text { even } \\
2,1 \leq i \leq n, i \text { even, } 1 \leq j \leq m, i \text { even } \\
3,1 \leq j \leq m-1, j \text { even; } 1 \leq i \leq n-1 \\
4,1 \leq j \leq m, j \text { odd } i=n
\end{array}\right. \\
c\left(x_{i}\right)=\left\{\begin{array}{l}
1,1 \leq i \leq n-1, i \text { even } \\
2,1 \leq i \leq n-1, i \text { odd } \\
3, i=n
\end{array}\right.
\end{gathered}
$$

It is easy to see that $c: V\left(W_{n} \odot P_{m}\right) \rightarrow\{1,2, \ldots, 3\}$ and $c: V\left(W_{n} \odot P_{m}\right) \rightarrow\{1,2, \ldots, 4\}$, for $n$ even and odd respectively, is proper coloring. Thus, $\chi\left(W_{n} \odot P_{m}\right)=3$ and $\chi\left(W_{n} \odot P_{m}\right)=4$, for $n$ even and odd respectively. By definition, since $\min \left\{|c(N(v))|\right.$, for every $\left.v \in V\left(W_{n} \odot P_{m}\right)\right\}=2$, it implies $\chi\left(W_{n} \odot P_{m}\right)=\chi_{d}\left(W_{n} \odot P_{m}\right)$. It completes the proof.

Problem 4. Let $G$ be a crown product of $W_{n}$ on $P_{m}$. For $n \geq 3$ dan $m \geq 2$, determine the $r$-dynamic chromatic number of $G$ when $r \geq 3$.

Theorem 1.9. Let $G$ be a crown product of $C_{n}$ on $S_{m}$. For $n \geq 3$ dan $m \geq 3$, the r-dynamic chromatic number of $G$ is

$$
\chi\left(C_{n} \odot S_{m}\right)=\chi_{d}\left(C_{n} \odot S_{m}\right)=\left\{\begin{array}{l}
3, \text { for } n \text { even } \\
4, \text { for } n \text { odd }
\end{array}\right.
$$


Proof. The graph $C_{n} \odot S_{m}$ is a connected graph with vertex set $V\left(C_{n} \odot S_{m}\right)=\{A\} \cup\left\{x_{j} ; 1 \leq\right.$ $j \leq m\} \cup\left\{y_{i} ; 1 \leq i \leq n\right\} \cup\left\{y_{i, j} ; 1 \leq i \leq n ; 1 \leq j \leq m\right\}$ and $E\left(C_{n} \odot S_{m}\right)=\left\{A x_{j} ; 1 \leq j \leq m\right\}$ $\cup\left\{A y_{i} ; 1 \leq i \leq n\right\} \cup\left\{x_{j} y_{i, j} ; 1 \leq i \leq n ; 1 \leq j \leq m\right\} \cup\left\{y_{i} y_{i+1} ; 1 \leq i \leq n-1\right\} \cup\left\{y_{n} y_{1}\right\}$ $\cup\left\{y_{i, j} y_{i+1, j} ; 1 \leq i \leq n-1 ; 1 \leq j \leq m\right\} \cup\left\{y_{n, j} y_{1, j} ; 1 \leq j \leq m\right\}$. Thus $\left|V\left(C_{n}\left[S_{m}\right]\right)\right|=n m+n+$ $m+1$ and $\left|E\left(C_{n} \odot S_{m}\right)\right|=2 n m+m+2 n$ and $\Delta\left(C_{n} \odot S_{m}\right)=m+n$. By Observation 1, the lower bound of $r$-dynamic chromatic number $\chi_{r}\left(C_{n} \odot S_{m}\right) \geq \min \left\{\Delta\left(C_{n} \odot S_{m}\right), r\right\}+1=\{m+n, r\}+1$. Define the vertex colouring $c: V\left(C_{n} \odot S_{m}\right) \rightarrow\{1,2, \ldots, k\}$ for $n \geq 3$ and $m \geq 3$ as follows: $A=1, c\left(x_{j}\right)=2,1 \leq j \leq m$ and

For $n$ even

$$
c\left(y_{i}\right)=\left\{\begin{array}{l}
2,1 \leq i \leq n, i \text { odd } \\
3,1 \leq i \leq n, i \text { even }
\end{array} ; c\left(y_{i, j}\right)=\left\{\begin{array}{l}
1,1 \leq i \leq n, i \text { odd }, 1 \leq j \leq m \\
3,1 \leq i \leq n, i \text { even, } 1 \leq j \leq m
\end{array}\right.\right.
$$

For $n$ odd

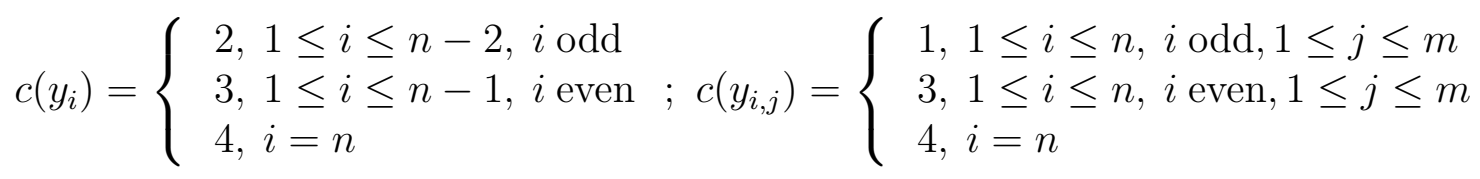

It is easy to see that $c: V\left(C_{n} \odot S_{m}\right) \rightarrow\{1,2, \ldots, 3\}$ and $c: V\left(C_{n} \odot S_{m}\right) \rightarrow\{1,2, \ldots, 4\}$, for $n$ even and odd respectively, is proper coloring. Thus, $\chi\left(C_{n} \odot S_{m}\right)=3$ and $\chi\left(C_{n} \odot S_{m}\right)=4$, for $n$ even and odd respectively. By definition, since $\min \left\{|c(N(v))|\right.$, for every $\left.v \in V\left(C_{n} \odot S_{m}\right)\right\}=$ $2 \leq \delta\left(C_{n} \odot S_{m}\right)=3$, it implies $\chi\left(C_{n} \odot S_{m}\right)=\chi_{d}\left(C_{n} \odot S_{m}\right)$. It completes the proof.

Problem 5. Let $G$ be a crown product of $C_{n}$ on $S_{m}$. For $n \geq 3$ dan $m \geq 3$, determine the $r$-dynamic chromatic number of $G$ when $r \geq 3$.

Theorem 1.10. Let $G$ be a shackle of cartesian product $P_{n}$ and $C_{m}$. For $n \geq 2$ and $m \geq 3$, the $r$-dynamic chromatic number of $G$ is

$$
\chi\left(\operatorname{shack}\left(P_{n} \square C_{m}, v, s\right)\right)=\chi_{d}\left(\operatorname{shack}\left(P_{n} \square C_{m}, v, s\right)\right)=\left\{\begin{array}{l}
3, \text { for } n \text { even } \\
4, \text { for } n \text { odd }
\end{array}\right.
$$

Proof. The shackle of cartesian product $P_{n}$ and $C_{m}$, denoted by $\operatorname{shack}\left(P_{n} \square C_{m}, v, s\right)$, is a connected graph with vertex set $V=\left\{x_{i, j}^{k} ; 1 \leq i \leq n ; 1 \leq j \leq m ; 1 \leq k \leq s\right\} \cup\left\{x_{n, j}^{k} ; 1 \leq j \leq\right.$ $m ; 1 \leq k \leq s\} \cup\left\{x_{n, j}^{r} ; 1 \leq j \leq m\right\}$ dan $E=\left\{x_{i, j}^{k} x_{i, j+1}^{k} ; 1 \leq i \leq n ; 1 \leq j \leq m-1 ; 1 \leq k \leq s\right\}$ $\cup\left\{x_{i, m}^{k} x_{i, 1}^{k} ; 1 \leq i \leq n ; 1 \leq k \leq s\right\} \cup\left\{x_{i, j}^{k} x_{i+1, j}^{k} ; 1 \leq i \leq n ; 1 \leq j \leq m ; 1 \leq k \leq s\right\}$. Thus $\left|V\left(\operatorname{shack}\left(P_{n} \square C_{m}, v, s\right)\right)\right|=n m s-s+1$ and $\left|E\left(\operatorname{shack}\left(P_{n} \square C_{m}, v, s\right)\right)\right|=2 n m s-n s$ and $\Delta\left(\operatorname{shack}\left(P_{n} \square C_{m}, v, s\right)\right)=6$. By Observation 1, the lower bound of $r$-dynamic chromatic number $\chi_{r}\left(\operatorname{shack}\left(P_{n} \square C_{m}, v, s\right)\right) \geq \min \left\{\Delta\left(\operatorname{shack}\left(P_{n} \square C_{m}, v, s\right)\right), r\right\}+1=\{6, r\}+1$. Define the vertex colouring $c: V\left(\operatorname{shack}\left(P_{n} \square C_{m}, v, s\right)\right) \rightarrow\{1,2, \ldots, k\}$ for $n \geq 3$ and $m \geq 3$ as follows:

For $m$ even

$$
\begin{aligned}
& c\left(x_{i, j}^{k}\right)=\left\{\begin{array}{l}
1,1 \leq j \leq m-1, j \text { odd, } k \text { odd and } i \text { odd } \\
2,1 \leq j \leq m, j \text { even, } k \text { odd and } i \text { odd }
\end{array}\right. \\
& c\left(x_{i, j}^{k}\right)=\left\{\begin{array}{l}
1,1 \leq j \leq m, j \text { even, } k \text { odd and } i \text { even } \\
2,1 \leq j \leq m-1, j \text { odd, } k \text { odd and } i \text { even }
\end{array}\right.
\end{aligned}
$$




$$
\begin{aligned}
& c\left(x_{i, j}^{k}\right)=\left\{\begin{array}{l}
1,1 \leq j \leq m-1, j \text { odd, } k \text { odd and } i=n \\
2,1 \leq j \leq m-1, j \text { even, } k \text { odd and } i=n
\end{array}\right. \\
& c\left(x_{i, j}^{k}\right)=\left\{\begin{array}{l}
1,1 \leq j \leq m-1, j \text { even, } k \text { even and } i \text { odd } \\
2,1 \leq j \leq m, j \text { odd, } k \text { even and } i \text { odd }
\end{array}\right. \\
& c\left(x_{i, j}^{k}\right)=\left\{\begin{array}{l}
1,1 \leq j \leq m-1, j \text { odd, } k \text { even and } i \text { even } \\
2,1 \leq j \leq m, j \text { even, } k \text { even and } i \text { even }
\end{array}\right. \\
& c\left(x_{i, j}^{k}\right)=\left\{\begin{array}{l}
1,1 \leq j \leq m-1, j \text { even, } k \text { even and } i=n \\
2,1 \leq j \leq m-1, j \text { odd, } k \text { even and } i=n
\end{array}\right.
\end{aligned}
$$

For $m$ odd

$$
\begin{aligned}
& c\left(x_{i, j}^{k}\right)=\left\{\begin{array}{l}
1,1 \leq j \leq m-2, j \text { even, } k \text { even and } i \text { odd } \\
2,1 \leq j \leq m-1, j \text { odd, } k \text { even and } i \text { odd } \\
3, j=m
\end{array}\right. \\
& c\left(x_{i, j}^{k}\right)=\left\{\begin{array}{l}
1,1 \leq j \leq m-2, j \text { odd, } k \text { odd and } i \text { even } \\
2,1 \leq j \leq m-1, j \text { even, } k \text { odd and } i \text { even } \\
3, j=m
\end{array}\right. \\
& c\left(x_{i, j}^{k}\right)=\left\{\begin{array}{l}
1,1 \leq j \leq m-2, j \text { even, } k \text { odd and } i=n \\
2,1 \leq j \leq m-1, j \text { odd, } k \text { odd and } i=n \\
3, j=m
\end{array}\right.
\end{aligned}
$$

It is easy to see that $c: V\left(\operatorname{shack}\left(P_{n} \square C_{m}, v, s\right)\right) \rightarrow\{1,2\}$ and $c: V\left(C_{n} \odot S_{m}\right) \rightarrow\{1,2,3\}$, for $m$ even and odd respectively, are proper coloring. Thus, $\chi\left(\operatorname{shack}\left(P_{n} \square C_{m}, v, s\right)\right)=2$ and $\chi\left(\operatorname{shack}\left(P_{n} \square C_{m}, v, s\right)\right)=3$, for $m$ even and odd respectively. By definition, since $\min \{|c(N(v))|$, for every $v \in$ $V\left(\operatorname{shack}\left(P_{n} \square C_{m}, v, s\right)\right\}$

$=1 \leq \delta\left(\operatorname{shack}\left(P_{n} \square C_{m}, v, s\right)\right)=3$, thus we only have $\chi\left(\operatorname{shack}\left(P_{n} \square C_{m}\right)\right)=2$ and $\chi\left(\operatorname{shack}\left(P_{n} \square C_{m}, v, s\right)\right)=$ 3 , for $m$ even and odd respectively. It completes the proof.

Problem 6. Let $G$ be a shackle of cartesian product $P_{n}$ and $C_{m}$. For $n \geq 2$ and $m \geq 3$, determine the $r$-dynamic chromatic number of $G$ when $r \geq 2$.

Theorem 1.11. Let $G$ be a shackle of joint $S_{n}$ and $P_{m}$. For $n \geq 3$ and $m \geq 2$, the $r$-dynamic chromatic number of $G$ is

$$
\chi\left(\operatorname{shack}\left(S_{n}+P_{m}, v, s\right)\right)=\chi_{d}\left(\operatorname{shack}\left(S_{n}+P_{m}, v, s\right)\right)=\chi_{3}\left(\operatorname{shack}\left(S_{n}+P_{m}, v, s\right)\right)=4
$$

Proof. The shackle of joint $S_{n}$ and $P_{m}$, denoted by $\operatorname{shack}\left(S_{n}+P_{m}, v, s\right)$, is a connected graph with vertex set $V=\left\{A_{k}, x_{1}^{k}, x_{i}^{k}, y_{j}^{k}, p ; 1 \leq i \leq n ; 1 \leq j \leq m ; 1 \leq k \leq s\right\}$ and $E=\left\{A_{k} x_{i}^{k} ; 1 \leq\right.$ $i \leq n-1 ; 1 \leq k \leq s\} \cup\left\{A_{k} x_{i}^{k+1} ; 1 \leq k \leq s\right\} \cup\left\{A_{s} p\right\} \cup\left\{y_{j}^{k} y_{j+1}^{k} ; 1 \leq j \leq m-1 ; 1 \leq k \leq s\right\}$ $\cup\left\{A^{k} y_{j}^{k} ; 1 \leq j \leq m ; 1 \leq k \leq s\right\} \cup\left\{x_{i}^{k} y_{j}^{k} ; 1 \leq i \leq n-1 ; 1 \leq j \leq m ; 1 \leq k \leq s\right\} \cup\left\{x_{1}^{k+1} y_{j}^{k} ; 1 \leq\right.$ $j \leq m ; 1 \leq k \leq s-1\} \cup\left\{p y_{j}^{s} ; 1 \leq j \leq m\right\}$. Thus $\left|V\left(\operatorname{shack}\left(S_{n}+P_{m}, v, s\right)\right)\right|=n r+m r+1$ and $\left|E\left(\operatorname{shack}\left(S_{n}+P_{m}, v, s\right)\right)\right|=2 n m s+n s+2 m s-s$ and $\Delta\left(\operatorname{shack}\left(S_{n}+P_{m}, v, s\right)\right)=6$. By Observation 1, the lower bound of $r$-dynamic chromatic number $\chi_{r}\left(\operatorname{shack}\left(S_{n}+P_{m}, v, s\right)\right) \geq$ 
$\min \left\{\Delta\left(\operatorname{shack}\left(S_{n}+P_{m}, v, s\right)\right), r\right\}+1=\{6, r\}+1$. Define the vertex coloring $c: V\left(\operatorname{shack}\left(S_{n}+\right.\right.$ $\left.\left.P_{m}, v, s\right)\right) \rightarrow\{1,2, \ldots, k\}$ for $n \geq 3$ and $m \geq 2$ as follows: $c\left(A^{k}\right)=4$

$$
\begin{aligned}
& c\left(x_{i}^{k}\right)=\{3,1 \leq i \leq n-1 ; 1 \leq k \leq s \\
& c\left(y_{i}^{k}\right)=\left\{\begin{array}{l}
1,1 \leq j \leq m, j \text { odd } ; 1 \leq k \leq s \\
2,1 \leq j \leq m, j \text { even } ; 1 \leq k \leq s
\end{array}\right.
\end{aligned}
$$

It is easy to see that $c: V\left(\operatorname{shack}\left(S_{n}+P_{m}, v, s\right)\right) \rightarrow\{1,2, \ldots, 4\}$ is proper coloring. Thus, $\chi\left(\operatorname{shack}\left(S_{n}+P_{m}, v, s\right)\right)$

$=4$. By definition, since $\min \left\{|c(N(v))|\right.$, for every $v \in V\left(\operatorname{shack}\left(S_{n}+P_{m}, v, s\right)\right\}=3$, it implies $\chi\left(\operatorname{shack}\left(S_{n}+P_{m}\right)\right)=\chi_{d}\left(\operatorname{shack}\left(S_{n}+P_{m}\right)\right)=\chi_{3}\left(\operatorname{shack}\left(S_{n}+P_{m}\right)\right)$. It completes the proof.

Problem 7. Let $G$ be a shackle of joint $S_{n}$ and $P_{m}$. For $n \geq 3$ and $m \geq 2$, determine the $r$-dynamic chromatic number of $G$ when $r \geq 4$.

\section{Conclusions}

We have studied the $r$-dynamic coloring of some graph operations. The results show for each graph operation, its $r$-dynamic chromatic number has not been obtained completely for all values of $r$, therefore we left them as open problems for the further study.

\section{Acknowledgement}

We gratefully acknowledge the support CGANT - University of Jember of year 2016.

\section{References}

[1] J.L. Gross, J. Yellen and P. Zhang, Handbook of Graph Theory, Second Edition, CRC Press, Taylor and Francis Group, 2014.

[2] S.J. Kim and W.J. Park, List dynamic coloring of sparse graphs,Combinatorial optimization and applications. Lect. Notes Comput. Sci. 6831 (Springer, 2011), 156162.

[3] S.J. Kim, S. J. Lee, and W.J. Park, Dynamic coloring and list dynamic coloring of planar graphs. Discrete Applied Math. 161 (2013), 22072212.

[4] S. Akbari, M. Ghanbari, S. Jahanbekam, On the dynamic chromatic number of graphs, Combinatorics and graphs. Contemp. Math. 531 (Amer. Math. Soc. 2010), 118.

[5] B. Montgomery, Dynamic Coloring of Graphs. Ph.D Dissertation, West Virginia University, 2001.

[6] H.J. Lai, B. Montgomery, and H. Poon, Upper bounds of dynamic chromatic number. Ars Combin. 68 (2003), 193201.

[7] S Jahanbekam, J Kim, O Suil, D.B. West, On r-dynamic Coloring of Graphs, 2014, In Press.

[8] R.L. Brooks, On colouring the nodes of a network. Proc.Cambridge Philos. Soc. 37 (1941), 194197. 